IMA Journal of Numerical Analysis (2017) 37, 1087-1103

doi: 10.1093/imanum/drw037

Advance Access publication on August 3, 2016

\title{
Banded, stable, skew-symmetric differentiation matrices of high order
}

\author{
ERNST HAIRER* \\ Section de mathématiques, Université de Genève, 2-4 rue du Lièvre, CH-1211 Genève 4, Switzerland \\ *Corresponding author: Ernst.Hairer@unige.ch \\ AND \\ ARIEH ISERLES \\ Department of Applied Mathematics and Theoretical Physics, University of Cambridge, \\ Wilberforce Road, Cambridge CB4 1LE, UK \\ ai@damtp.cam.ac.uk
}

[Received on 9 November 2015; revised on 20 February 2016]

\begin{abstract}
Differentiation matrices play an important role in the space discretization of first-order partial differential equations. This work considers grids on a finite interval and treats homogeneous Dirichlet boundary conditions. Differentiation matrices of orders up to 6 are derived that are banded, stable and skew symmetric. To achieve these desirable properties, nonequidistant grids are considered.
\end{abstract}

Keywords: skew-symmetric differentiation matrices; order conditions; construction of differentiation matrices.

\section{Introduction}

This article deals with the approximation of the first derivative $u^{\prime}(\xi)$ on a grid over a finite interval

$$
a=\xi_{0}<\xi_{1}<\cdots<\xi_{n}<\xi_{n+1}=b .
$$

To keep the notation simple, we suppress the dependence of $\xi_{k}$ on $n$, the number of internal grid points. We always assume that the grid is dense in $[a, b]$, meaning that $\max _{k=0, \ldots, n}\left|\xi_{k+1}-\xi_{k}\right|=\mathcal{O}\left(n^{-1}\right)$. In view of applications to the space discretization of partial differential equations with Dirichlet boundary conditions, our main interest is in functions $u(\xi)$ vanishing at both endpoints. We assume that the derivative approximation is obtained by a linear combination of function values over the same grid. This leads to the consideration of

$$
u^{\prime}\left(\xi_{m}\right) \approx \sum_{k=1}^{n} \widehat{\mathcal{D}}_{m, k} u\left(\xi_{k}\right)
$$

for $m=1, \ldots, n$. The matrix $\left(\widehat{\mathcal{D}}_{m, k}\right)$ is called a differentiation matrix. Our interest is in such matrices that have the following features:

- The matrix $\left(\widehat{\mathcal{D}}_{m, k}\right)_{m, k=1}^{n}$ is banded, i.e., there exists an integer $r \geq 1$ such that $\widehat{\mathcal{D}}_{m, k}=0$ for $|m-k| \geq$ $r+1$. This assumption brings an evident computational advantage and guarantees that the derivative approximation is local. 
- The differentiation formula is stable, which means that the entries of the differentiation matrix satisfy $\left|\widehat{\mathcal{D}}_{m, k}\right|=\mathcal{O}(n)$.

- The approximation is of order $P \geq 2$. This means that for smooth functions $u(\xi)$ the defect in (1.2) is $\mathcal{O}\left(h^{P}\right)$, where $h=\max _{k=0}^{n}\left|\xi_{k+1}-\xi_{k}\right|$. For banded, stable grids this is equivalent to requiring that the formula (1.2) is exact for all polynomials of degree $\leq P$ that vanish at the endpoints.

- The matrix $\left(\widehat{\mathcal{D}}_{m, k}\right)_{m, k=1}^{n}$ is skew symmetric. Since the first derivative is a skew-symmetric operator, this is a natural assumption in the spirit of geometric numerical integration. It has several interesting implications for the discretization of partial differential equations (see Hairer \& Iserles, 2016).

For an equidistant grid $\left\{\xi_{k}=a+k h\right\}$ with $h=(b-a) /(n+1)$, the discretization

$$
u^{\prime}(\xi) \approx \frac{1}{2 h}(u(\xi+h)-u(\xi-h))
$$

leads to a differentiation matrix that satisfies all four properties, but which is only of second order. The nonzero entries are $\widehat{\mathcal{D}}_{k, k+1}=1 /(2 h)$ and $\widehat{\mathcal{D}}_{k, k-1}=-1 /(2 h)$. Our focus is on differentiation matrices of order higher than 2.

On an equidistant grid, the fourth-order approximation

$$
u^{\prime}(\xi) \approx \frac{1}{12 h}(u(\xi-2 h)-8 u(\xi-h)+8 u(\xi+h)-u(\xi+2 h))
$$

can be used at all grid points with the exception of $\xi_{1}$ and $\xi_{n}$. For these two grid points, one-sided approximations can be used. This, however, destroys the skew symmetry of the matrix.

The difficulty in the construction of skew-symmetric differentiation matrices lies in the fact that we are dealing with Dirichlet boundary conditions on a finite interval. For equidistant infinite grids on the whole real line or for equidistant finite grids and periodic boundary conditions, a differentiation formula like (1.3) can be used everywhere, retaining skew symmetry.

Finite difference approximations satisfying a 'summation by parts rule' (see Kreiss \& Scherer, 1974; Strand, 1994; Olsson, 1995) are closely connected to this work. Their approach consists of using an equidistant grid and a standard differentiation formula (like (1.3)) in the interior of the interval. Close to the endpoints, one-sided finite difference approximations are considered such that the differentiation matrix becomes skew symmetric with respect to a modified scalar product. Under the assumption that the modified scalar product is defined by a diagonal (positive definite) matrix, approximations of order $\tau$ close to the boundary and of order $2 \tau$ in the interior are proposed for $\tau=1, \ldots, 4$. For scalar products with a full matrix at the endpoints of the interval, difference approximations with order $\tau=3$ and $\tau=5$ at the boundary and order $\tau+1$ in the interior have been computed. Related work for arbitrary fixed grids can be found in McLachlan (2003) and Kitson et al. (2003).

In this work we propose an alternative to the 'summation by parts' approach. We do not modify the inner product in the discrete summation by parts formula, but we consider instead a grid that is nonuniform near the endpoints of the interval. This gives similar results concerning the bandwidth of the differentiation matrices in the interior of the interval and concerning the order close to the boundary. It seems that higher orders can be obtained with the present approach. It is well known that these approaches yield stable finite difference space discretizations for hyperbolic systems. The absence of 'skew symmetry' can lead to unstable space discretizations. This article is restricted to homogeneous boundary conditions and to 
approximations of the first space derivative. We expect that an extension to nonhomogeneous boundary conditions or to Neumann boundary conditions will give similar results.

The structure of the considered differentiation matrices and that of the underlying grid are presented in Section 2. The following Section 3 is devoted to conditions on the grid that are necessary for the existence of higher-order skew-symmetric differentiation matrices. The construction of such matrices is detailed in Section 4 for order 3, in Section 5 for order 4 and higher orders (up to order 6) are discussed in Section 6.

\section{Structure of considered differentiation matrices}

The differentiation matrix originating in a differentiation formula such as (1.3) is skew symmetric everywhere with the exception of its left upper and right lower corners. The idea is to modify the coefficients in these parts to achieve skew symmetry and retain high order, stability and the banded structure. It follows from the results of Iserles (2014) and Hairer \& Iserles (2016) that this is not possible for an equidistant grid. We therefore modify the grid, but we do this only close to the endpoints of the interval.

\subsection{The choice of the grid}

We fix positive integers $N$ and $L$ and consider the symmetric grid

$$
x_{k}=\left\{\begin{array}{clrl}
-a_{-k} h, & k & =-L, \ldots,-1, \\
k h, & k & =0,1, \ldots, N, \\
1+a_{k-N} h, & k & =N+1, \ldots, N+L,
\end{array}\right.
$$

where $h=1 / N$ and $a_{1}, \ldots, a_{L}$ are parameters $\left(0<a_{1}<\cdots<a_{L}\right)$, which may depend on $h$. This grid corresponds to the interval $\left[-a_{L} h, 1+a_{L} h\right]$ and has $n=N+2 L-1$ interior grid points. It is equidistant except for a few subintervals at the endpoints.

\subsection{The pattern of the differentiation matrix}

Associated to the grid (2.1), we consider differentiation matrices $\left(\mathcal{D}_{m, k}\right)$ yielding approximations

$$
u^{\prime}\left(x_{m}\right) \approx \sum_{k=-L+1}^{N+L-1} \mathcal{D}_{m, k} u\left(x_{k}\right)
$$

for functions $u(x)$ vanishing at the endpoints $x_{-L}$ and $x_{N+L}$. For the definition of the matrix $\left(\mathcal{D}_{m, k}\right)$, we consider a basic differentiation rule for an equidistant grid

$$
u^{\prime}(x) \approx \sum_{k=-R}^{R} \delta_{k} u(x+k h)
$$

satisfying $\delta_{0}=0$ and $\delta_{-k}=-\delta_{k}$. For example, the differentiation rule (1.3) has $R=2, \delta_{1}=8 /(12 h)$ and $\delta_{2}=-1 /(12 h)$. In general,

$$
\delta_{k}=\frac{(-1)^{k-1}}{k h} \frac{R !^{2}}{(R-k) !(R+k) !} \quad k=1, \ldots, R
$$




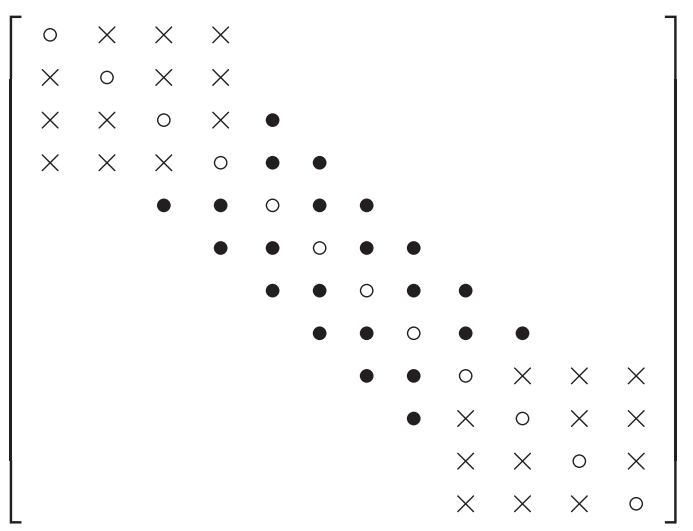

FIG. 1. Pattern of the differentiation matrix with $L=3, M=2, R=2$ and $N=7$.

gives rise to an $(2 R)$-order differentiation rule.

In addition to the integers $N$ and $L$ of Section 2.1, we fix a positive integer $M$. We then assume that

$$
\mathcal{D}_{m, k}=\delta_{k-m}
$$

for indices $m, k$ satisfying $|k-m| \leq R$ and $M \leq m \leq N-M$ or $M \leq k \leq N-M$. The remaining entries are zero, with the exception of the two $(M+L-1) \times(M+L-1)$ matrices on the upper left and lower right corners: $\left(\mathcal{D}_{m, k}\right)_{m, k=-L+1}^{M-1}$ and $\left(\mathcal{D}_{m, k}\right)_{m, k=N-M+1}^{N+L-1}$. We assume them to be skew symmetric. Moreover, we assume that the whole matrix is skew persymmetric, so that the lower right sub-matrix is determined by the upper left one via

$$
\mathcal{D}_{N-m, N-k}=-\mathcal{D}_{m, k} \quad \text { for } \quad-L+1 \leq m, k \leq M-1 \text {. }
$$

The whole situation is illustrated in Fig. 1 for $L=3, M=2, R=2$ and $N=7$. The symbol $\bullet$ indicates the entries given by (2.5) and $\times$ indicates nonzero entries of the two blocks.

For example, if we consider the differentiation formula (1.3) as basic rule, we have

$$
\mathcal{D}_{m, k}=\left\{\begin{array}{cl}
1 /(12 h) & \text { if } k=m-2 \\
-8 /(12 h) & \text { if } k=m-1 \\
0 & \text { if } k=m \\
8 /(12 h) & \text { if } k=m+1 \\
-1 /(12 h) & \text { if } k=m+2
\end{array}\right.
$$

for indices $m, k$ satisfying $M \leq m \leq N-M$ or $M \leq k \leq N-M$. For smooth functions $u(x)$, the defect in (2.2) is $\mathcal{O}\left(h^{4}\right)$ for $M \leq m \leq N-M$. Our aim is to complete the matrix $\mathcal{D}$ in the upper left and lower right corners such that $\mathcal{D}$ is skew symmetric, and for all indices the defect in (2.2) is at least $\mathcal{O}\left(h^{3}\right)$.

We note that the differentiation matrices considered here are computationally very efficient. For a given basic differentiation rule only a finite, fairly small number of coefficients (independent of the number of grid points) need to be computed. 


\subsection{Transformation to an arbitrary grid}

The grid (2.1) is convenient for theoretical investigations. It has $n=N+2 L-1$ interior grid points. In practice we have to work with a grid (1.1) for an interval $[a, b]$. We connect both grids by

$$
\xi_{k}=a+\eta\left(x_{k-L}+a_{L} h\right) \quad k=0,1, \ldots, n+1, \quad \text { where } \quad \eta=\frac{b-a}{1+2 a_{L} h} .
$$

Note that $\eta$ is the ratio between the lengths of the corresponding intervals. The grid $\left(\xi_{k}\right)$ is also essentially equidistant, and only a few subintervals at the end points have a different length.

The coefficients of the differentiation matrix (1.2) have to be scaled by $\eta$ :

$$
\widehat{\mathcal{D}}_{m, k}=\eta^{-1} \mathcal{D}_{m, k} .
$$

\section{Order conditions}

A banded, stable differentiation matrix is of order $P$ if, for all $m$, the relation (2.2) is satisfied exactly for all polynomials $u(x)$ of degree $P$ vanishing at the endpoints.

\subsection{The linear system for the order conditions}

For differentiation matrices as defined in Section 2.2, the order conditions for order $P$ lead to a linear system with:

- $K(K-1) / 2$ unknowns, where $K=M+L-1$ : these are the elements below the diagonal of the skew-symmetric sub-matrix $\left(\mathcal{D}_{m, k}\right)_{m, k=-L+1}^{M-1}$.

- $\quad(P-1) K$ equations: these are obtained by imposing equality in (2.2) for $m=-L+1, \ldots, M-1$ and for polynomials $u(x)$ vanishing at $-a_{L} h$ and at $1+a_{L} h$. Such polynomials are of the form $u(x)=\left(a_{L} h\left(1+a_{L} h\right)+x(1-x)\right) p(x)$, where $p$ is of degree $P-2$.

The resulting linear system can be written in the form

$$
\mathcal{A}_{L, M} \mathcal{D}_{L, M}=\mathbf{b}_{L, M},
$$

where $\mathcal{D}_{L, M}$ is a linear arrangement of the subdiagonal elements of the $K \times K$ block $\left(\mathcal{D}_{m, k}\right)_{m, k=-L+1}^{M-1}$.

For example, for $L=3$ and $M=2$ we have

$$
\mathcal{D}_{L, M}=\left(\mathcal{D}_{-1,-2}, \mathcal{D}_{0,-2}, \mathcal{D}_{0,-1}, \mathcal{D}_{1,-2}, \mathcal{D}_{1,-1}, \mathcal{D}_{1,0}\right)
$$

and the matrix $\mathcal{A}_{L, M}$ is given (for $P=3$ ) by

$$
\mathcal{A}_{L, M}=\left(\begin{array}{cccccc}
-u^{1}\left(x_{-1}\right) & -u^{1}(0) & 0 & -u^{1}(h) & 0 & 0 \\
u^{1}\left(x_{-2}\right) & 0 & -u^{1}(0) & 0 & -u^{1}(h) & 0 \\
0 & u^{1}\left(x_{-2}\right) & u^{1}\left(x_{-1}\right) & 0 & 0 & -u^{1}(h) \\
0 & 0 & 0 & u^{1}\left(x_{-2}\right) & u^{1}\left(x_{-1}\right) & u^{1}(0) \\
-u^{2}\left(x_{-1}\right) & -u^{2}(0) & 0 & -u^{2}(h) & 0 & 0 \\
u^{2}\left(x_{-2}\right) & 0 & -u^{2}(0) & 0 & -u^{2}(h) & 0 \\
0 & u^{2}\left(x_{-2}\right) & u^{2}\left(x_{-1}\right) & 0 & 0 & -u^{2}(h) \\
0 & 0 & 0 & u^{2}\left(x_{-2}\right) & u^{2}\left(x_{-1}\right) & u^{2}(0)
\end{array}\right),
$$


where $u^{j}(x)=\left(a_{L} h\left(1+a_{L} h\right)+x(1-x)\right) p^{j}(x)$, while $p^{1}$ and $p^{2}$ are linearly independent polynomials of degree $P-2=1$.

The vector $\mathbf{b}_{L, M}$ consists of $P-1$ sub-vectors $\mathbf{b}_{L, M}^{j}$ (for $j=1, \ldots, P-1$ ), each of which is formed by the $K$ elements $(m=-L+1, \ldots, M-1)$

$$
\left(u^{j}\right)^{\prime}\left(x_{m}\right)-\sum_{k \geq M} \mathcal{D}_{m, k} u^{j}\left(x_{k}\right)
$$

The coefficients $\mathcal{D}_{m, k}=\delta_{k-m}$ are those inherited from the basic differentiation rule (2.4). Since $\delta_{k-m}=0$ for $k-m>R$, the sum in (3.2) is nonzero only for $m \geq M-R$.

LEMMA 3.1 For a differentiation matrix of order $P \geq 2$, the row rank of the matrix $\mathcal{A}_{L, M}$ is at most

$$
(P-1) K-\left(\begin{array}{l}
P \\
2
\end{array}\right)
$$

In particular, it is $2 K-3$ for $P=3$ and $3 K-6$ for $P=4$.

Proof. We let $\mathbf{u}^{j}=\left(u^{j}\left(x_{-L+1}\right), \ldots, u^{j}\left(x_{M-1}\right)\right)$, and set $\mathbf{0}=(0, \ldots, 0)$.

Case $P=3$. Multiplication of $\mathcal{A}_{L, M}$ from the left with one of the $2 K$-dimensional row vectors $\left(\mathbf{u}^{1}, \mathbf{0}\right)$, $\left(\mathbf{0}, \mathbf{u}^{2}\right)$ and $\left(\mathbf{u}^{2}, \mathbf{u}^{1}\right)$ yields the zero vector. Since these vectors are linearly independent, this proves the statement for $P=3$.

Case $P=4$. In this case we multiply $\mathcal{A}_{L, M}$ from the left with one of the $3 K$-dimensional row vectors $\left(\mathbf{u}^{1}, \mathbf{0}, \mathbf{0}\right),\left(\mathbf{0}, \mathbf{u}^{2}, \mathbf{0}\right),\left(\mathbf{0}, \mathbf{0}, \mathbf{u}^{3}\right)$, and $\left(\mathbf{u}^{2}, \mathbf{u}^{1}, \mathbf{0}\right),\left(\mathbf{u}^{3}, \mathbf{0}, \mathbf{u}^{1}\right),\left(\mathbf{0}, \mathbf{u}^{3}, \mathbf{u}^{2}\right)$ to obtain the zero vector. This proves the case $P=4$.

A straightforward extension yields the statement for the general case.

\subsection{Necessary conditions for the grid}

Lemma 3.1 leads to necessary conditions for achieving order $P$. We denote by $\mathbf{u}_{L, M}^{j}=\left(u^{j}\left(x_{-L+1}\right), \ldots\right.$, $\left.u^{j}\left(x_{M-1}\right)\right)$ the vectors used in the proof of Lemma 3.1, and we split the vector $\mathbf{b}_{L, M}$ into $\left(\mathbf{b}_{L, M}^{1}, \ldots, \mathbf{b}_{L, M}^{P-1}\right)$. We then have the following necessary order conditions for the existence of a solution for (3.1):

$$
\mathbf{u}_{L, M}^{j} \cdot \mathbf{b}_{L, M}^{k}+\mathbf{u}_{L, M}^{k} \cdot \mathbf{b}_{L, M}^{j}=0 \quad \text { for } \quad 1 \leq j \leq k \leq P-1 .
$$

The dot denotes the scalar product of two vectors. These relations represent nonlinear conditions for the parameters $a_{1}, \ldots, a_{L}$.

LEMMA 3.2 Assume that the basic differentiation rule (2.4) is exact for all polynomials of degree $P$, then the necessary conditions (3.3) are the same for all $M \geq R$. 
Proof. We consider the first expression of the left-hand side of (3.3) and compute the difference for two consecutive values of $M$. With the convention $\delta_{j}=0$ for $|j|>R$, this yields

$$
\begin{aligned}
\mathbf{u}_{L, M+1}^{j} & \cdot \mathbf{b}_{L, M+1}^{k}-\mathbf{u}_{L, M}^{j} \cdot \mathbf{b}_{L, M}^{k} \\
= & \sum_{m=-L+1}^{M} u^{j}\left(x_{m}\right)\left(\left(u^{k}\right)^{\prime}\left(x_{m}\right)-\sum_{l \geq M+1} \delta_{l-m} u^{k}\left(x_{l}\right)\right) \\
& -\sum_{m=-L+1}^{M-1} u^{j}\left(x_{m}\right)\left(\left(u^{k}\right)^{\prime}\left(x_{m}\right)-\sum_{l \geq M} \delta_{l-m} u^{k}\left(x_{l}\right)\right) \\
= & u^{j}\left(x_{M}\right)\left(\left(u^{k}\right)^{\prime}\left(x_{M}\right)-\sum_{l \geq M+1} \delta_{l-M} u^{k}\left(x_{l}\right)\right)+\sum_{m=-L+1}^{M-1} u^{j}\left(x_{m}\right) \delta_{M-m} u^{k}\left(x_{M}\right) .
\end{aligned}
$$

Adding the same expression with exchanged values of $j$ and $k$, and using the skew symmetry relation $\delta_{-j}=-\delta_{j}$ gives

$$
\begin{gathered}
\left(\mathbf{u}_{L, M+1}^{j} \cdot \mathbf{b}_{L, M+1}^{k}+\mathbf{u}_{L, M+1}^{k} \cdot \mathbf{b}_{L, M+1}^{j}\right)-\left(\mathbf{u}_{L, M}^{j} \cdot \mathbf{b}_{L, M}^{k}+\mathbf{u}_{L, M}^{k} \cdot \mathbf{b}_{L, M}^{j}\right) \\
=u^{j}\left(x_{M}\right)\left(\left(u^{k}\right)^{\prime}\left(x_{M}\right)-\sum_{|l-M| \leq R} \delta_{l-M} u^{k}\left(x_{l}\right)\right) \\
\quad+u^{k}\left(x_{M}\right)\left(\left(u^{j}\right)^{\prime}\left(x_{M}\right)-\sum_{|l-M| \leq R} \delta_{l-M} u^{j}\left(x_{l}\right)\right) .
\end{gathered}
$$

For $M \geq R$, the grid points $x_{l}$ for $l=M-R, \ldots, M+R$ all belong to the equidistant sub-grid of (2.1). Since the degree of the polynomials $u^{j}$ and $u^{k}$ is $\leq P$, our assumption implies that the right-hand side of the above equation vanishes identically.

As a consequence of Lemma 3.2, there is no advantage in considering the nonlinear system (3.3) for $M$ larger than $R$. We therefore always assume $M=R$.

Lemma 3.3 The solutions of the nonlinear system (3.3) are independent of the choice of the functions $p^{j}(x)$ in the definition of $u^{j}(x)$.

Proof. If $\hat{u}^{j}$ (for $j=1, \ldots, P-1$ ) is a linear combination of the functions $u^{j}, j=1, \ldots, P-1$, the expressions in (3.3) for the hat quantities are a linear combination of those for the original functions. Consequently, the set of solutions remains unchanged.

\subsection{An explicit form of the order conditions}

With the functions $u^{j}(x)=\left(a_{L} h\left(1+a_{L} h\right)+x(1-x)\right) x^{j-1}$ and the notation $A=a_{L}\left(1+a_{L} h\right)$, the condition (3.3) for order $P$ can be written as

$$
\sum_{m=-L+1}^{M-1}\left(u^{j}\left(x_{m}\right)\left(u^{k}\right)^{\prime}\left(x_{m}\right)+u^{k}\left(x_{m}\right)\left(u^{j}\right)^{\prime}\left(x_{m}\right)\right)=Q_{j, k}(A)
$$


for $1 \leq j \leq k \leq P-1$, where $Q_{j, k}(A)=P_{j, k}(A)+P_{k, j}(A)$ and

$$
P_{j, k}(A)=\sum_{m=M-R}^{M-1} u^{j}(m h) \sum_{l=M}^{m+R} \delta_{l-m} u^{k}(l h)
$$

We have exploited the fact that $\delta_{l-m}=0$ for $|l-m|>R$ and that $x_{m}=m h$ for $m \geq 0$. The derivative of $u^{j}(x)=(h A+x(1-x)) x^{j-1}$ is

$$
\left(u^{j}\right)^{\prime}(x)=\left((j-1) h A+j x-(j+1) x^{2}\right) x^{j-2} .
$$

Using the abbreviation (with the convention $0^{0}=1$ )

$$
S_{k}=\sum_{l=-L+1}^{M-1} x_{l}^{k}=h^{k}\left(\left(-a_{L-1}\right)^{k}+\ldots+\left(-a_{1}\right)^{k}+0^{k}+1^{k}+\ldots+(M-1)^{k}\right),
$$

the order condition (3.4) thus becomes

$$
\begin{aligned}
& h^{2} A^{2}(j+k-2) S_{j+k-3}+2 h A\left((j+k-1) S_{j+k-2}-(j+k) S_{j+k-1}\right) \\
& \quad+\left((j+k) S_{j+k-1}-2(j+k+1) S_{j+k}+(j+k+2) S_{j+k+1}\right)=Q_{j, k}(A) .
\end{aligned}
$$

We note that, not only $S_{k}$ contains the factor $h^{k}$, but also $u^{j}\left(x_{m}\right)$ and $u^{k}\left(x_{l}\right)$ contain the factor $h^{j}$ and $h^{k}$, respectively. Therefore, we can divide the equation by $h^{j+k-1}$. Doing this and using the notation

$$
s_{k}=\left(-a_{L-1}\right)^{k}+\ldots+\left(-a_{1}\right)^{k}+0^{k}+1^{k}+(M-1)^{k}
$$

the order conditions finally become (for $1 \leq j \leq k \leq P-1$ )

$$
\begin{aligned}
& A^{2}(j+k-2) s_{j+k-3}+2 A\left((j+k-1) s_{j+k-2}-(j+k) h s_{j+k-1}\right) \\
& \quad+\left((j+k) s_{j+k-1}-2(j+k+1) h s_{j+k}+(j+k+2) h^{2} s_{j+k+1}\right)=q_{j, k}^{h}(A),
\end{aligned}
$$

where $q_{j, k}^{h}(A)=h^{-j-k+1} Q_{j, k}(A)$.

REMARK 3.4 The left-hand expression of (3.7) is seen to depend only on the sum $j+k$. Therefore, it is necessary that $q_{j, k}^{h}(A)=q_{\hat{j}, \hat{k}}^{h}(A)$, whenever $j+k=\hat{j}+\hat{k}$. For $P=3$ this does not give any restriction. For order $P=4$ we get a single condition, $q_{2,2}^{h}(A)=q_{1,3}^{h}(A)$. For order $P=5$ there are two additional conditions $q_{2,3}^{h}(A)=q_{1,4}^{h}(A)$ and $q_{3,3}^{h}(A)=q_{2,4}^{h}(A)$, and for general $P$ there is a total of $\left(\begin{array}{c}P-2 \\ 2\end{array}\right)$ conditions. 


\subsection{Connection with the necessary conditions of Hairer \& Iserles (2016)}

For curiosity, we relate the order conditions (3.3) to those obtained in Hairer \& Iserles (2016). Without any stability restriction on the differentiation matrix, it is shown in Hairer \& Iserles (2016) that the grid of a $P$ th order skew-symmetric differentiation matrix has to satisfy

$$
\sum_{k=-L+1}^{N+L-1} f^{\prime}\left(x_{k}\right)=0 \quad \text { for } \quad f(x)=\left(x-x_{-L}\right)^{2}\left(x_{N+L}-x\right)^{2} p(x)
$$

for all polynomials $p(x)$ of degree $2 P-4$. For the case of a symmetric grid, the condition (3.8) is automatically fulfilled for polynomials satisfying $p(1-x)=p(x)$, so that only the polynomials $p(x)=(x-1 / 2)^{2 j-1}, j=1, \ldots, P-2$ have to be considered. For $P=3$, this is one equation in contrast to Condition (3.3) which consists of three equations for $P=3$. This shows that additional order conditions have to be satisfied for grids of the form (2.1).

LEMMA 3.5 If a grid (2.1) satisfies the order conditions (3.3) for order $P$, then it also satisfies the condition (3.8) for the same order $P$.

Proof. We put $u(x)=\left(x-x_{-L}\right)\left(x_{N+L}-x\right)(x-1 / 2)^{j-1} x$ for $j \in\{1, \ldots, P-2\}$ and let $v(x)=u(1-x)$, so that for the function $f(x)$ of (3.8) we have

$$
f(x)=\left(x-x_{-L}\right)^{2}\left(x_{N+L}-x\right)^{2}\left(x-\frac{1}{2}\right)^{2 j-1}=\frac{1}{2}\left(u(x)^{2}-v(x)^{2}\right) .
$$

Condition (3.3) with $j=k$ and $u(x)$, respectively, $v(x)$, in place of $u^{j}(x)$ (this is justified by Lemma 3.3) thus reads

$$
\begin{aligned}
& \sum_{k=-L+1}^{M-1} u\left(x_{k}\right)\left(u^{\prime}\left(x_{k}\right)-\sum_{j \geq M} \mathcal{D}_{k, j} u\left(x_{j}\right)\right)=0 \\
& \sum_{k=-L+1}^{\widehat{M}-1} v\left(x_{k}\right)\left(v^{\prime}\left(x_{k}\right)-\sum_{j \geq \widehat{M}} \mathcal{D}_{k, j} v\left(x_{j}\right)\right)=0,
\end{aligned}
$$

where $M \geq R$ and $\widehat{M} \geq R$ can be arbitrarily fixed (Lemma 3.2). Using the symmetry of the grid and the relation $v(x)=u(1-x)$, the condition for $v(x)$ can be written in terms of $u(x)$ as:

$$
\sum_{k=N-\widehat{M}+1}^{N+L-1} u\left(x_{k}\right)\left(-u^{\prime}\left(x_{k}\right)-\sum_{j \leq N-\widehat{M}} \mathcal{D}_{N-k, N-j} u\left(x_{j}\right)\right)=0 .
$$

By the central skew symmetry $\mathcal{D}_{N-k, N-j}=-\mathcal{D}_{k, j}$ and with the choice of $\widehat{M}$ such that $M+\widehat{M}=N+1$, a subtraction of both relations for $u(x)$ yields

$$
\sum_{k=-L+1}^{N+L-1} u\left(x_{k}\right) u^{\prime}\left(x_{k}\right)=0
$$


because the two double sums cancel by the skew symmetry of $\mathcal{D}_{k, j}$. An analogous relation is obtained for $v(x)$. The statement now follows from (3.9), and the fact that the relation (3.8) is satisfied for $u(x)^{2}$ and $v(x)^{2}$ by (3.10).

This is a point to emphasize a subtle issue. In Hairer \& Iserles (2016), the condition (3.8) is also sufficient for the existence of a skew-symmetric matrix of order $P$ on a given grid, except that such a matrix, which is banded, need not be stable. Once we construct differentiation matrices like in this article, (3.8) is clearly insufficient. It is an open problem whether (3.8) is necessary and sufficient for the construction of a stable, skew-symmetric differentiation matrix for every $P \geq 3$.

\section{Differentiation matrices of order 3}

In this section, we construct skew-symmetric differentiation matrices of the form introduced in Section 2 and based on the differentiation formula (1.3).

\subsection{Order conditions for order 3}

For the choice (2.6) of the basic differentiation formula we have $R=2$. We put $M=2$ and get $q_{j, k}^{h}(A)=p_{j, k}^{h}(A)+p_{k, j}^{h}(A)$ with (see Section 3.3)

$$
p_{j, k}^{h}(A)=\frac{1}{12 h^{j+k}}\left(-u^{j}(0) u^{k}(2 h)+u^{j}(h)\left(8 u^{k}(2 h)-u^{k}(3 h)\right)\right) .
$$

Inserting $u^{j}(x)=(h A+x(1-x)) x^{j-1}$ into (3.7) results in the system

$$
\begin{gathered}
2 A\left(s_{0}-2 h s_{1}\right)+\left(2 s_{1}-6 h s_{2}+4 h^{2} s_{3}\right)=q_{1,1}^{h}(A) \\
=\frac{1}{6}\left(6 A^{2}+18 A+13-36 h-26 h A+23 h^{2}\right) \\
A^{2} s_{0}+2 A\left(2 s_{1}-3 h s_{2}\right)+\left(3 s_{2}-8 h s_{3}+5 h^{2} s_{4}\right)=q_{1,2}^{h}(A) \\
=\frac{1}{6}\left(9 A^{2}+26 A+18-48 h-36 h A+30 h^{2}\right) \\
2 A^{2} s_{1}+2 A\left(3 s_{2}-4 h s_{3}\right)+\left(4 s_{3}-10 h s_{4}+6 h^{2} s_{5}\right)=q_{2,2}^{h}(A) \\
=\frac{1}{6}\left(13 A^{2}+36 A+23-60 h-50 h A+37 h^{2}\right)
\end{gathered}
$$

which is necessary for order $P=3$. We choose $L=3$, so that three parameters $a_{1}, a_{2}$ and $a_{3}$ are available. We solve this system numerically and obtain the following values:

\begin{tabular}{r|ccc}
\hline$N$ & $a_{1}$ & $a_{2}$ & $a_{3}$ \\
\hline 10 & 1.003781632659 & 2.007632287816 & 2.752472830434 \\
100 & 1.003023528871 & 2.004132100779 & 2.762836922708 \\
1000 & 1.002922802068 & 2.003722837122 & 2.764655844052 \\
10000 & 1.002912404585 & 2.003681363399 & 2.764850531480 \\
100000 & 1.002911361492 & 2.003677210713 & 2.764870136085 \\
$\infty$ & 1.002911245556 & 2.003676749244 & 2.764872315908 \\
\hline
\end{tabular}


The coefficients of the corresponding differentiation matrix are obtained from the solution of the linear system (3.1). Their values, multiplied by $h$, are as follows:

\begin{tabular}{c|ccc}
\hline$N$ & $\mathcal{D}_{-1,-2} / \mathcal{D}_{1,-2}$ & $\mathcal{D}_{0,-2} / \mathcal{D}_{1,-1}$ & $\mathcal{D}_{0,-1} / \mathcal{D}_{1,0}$ \\
\hline 10 & -0.3387467173411 & -0.4225146995560 & -0.3192512422566 \\
& 0.2032627246264 & -0.10262241060990 & -0.6027828536519 \\
100 & -0.3075432630829 & -0.4243406234660 & -0.3351340348980 \\
& 0.1896736705106 & -0.08174504699602 & -0.6135039576113 \\
1000 & -0.3034984572193 & -0.4242812535380 & -0.3374683924251 \\
& 0.1879789457053 & 0.07914665533779 & -0.6147792562106 \\
10000 & -0.3030820398568 & -0.4242713002458 & -0.3377122271803 \\
& 0.1878052095303 & 0.07888039374437 & -0.6149091785254 \\
$\infty$ & -0.3030356334373 & -0.4242701465998 & -0.3377394411464 \\
& 0.1877858563381 & 0.07885073516130 & -0.6149236416578 \\
\hline
\end{tabular}

All coefficients $\mathcal{D}_{m, k}$ are seen to be bounded by $\mathcal{O}\left(h^{-1}\right)$, and thus lead to stable discretizations.

\subsection{The limit case $h \rightarrow 0$}

We set $h=0$ in the order conditions (4.1) and we note that $A=a_{L}\left(1+a_{L} h\right)$ becomes $a_{L}$. The conditions (for order $P=3$ ) are thus (we write $a$ instead of $a_{L}$ )

$$
\begin{aligned}
2 a s_{0}+2 s_{1} & =q_{1,1}(a)=\frac{1}{6}\left(6 a^{2}+18 a+13\right) \\
a^{2} s_{0}+4 a s_{1}+3 s_{2} & =q_{1,2}(a)=\frac{1}{6}\left(9 a^{2}+26 a+18\right) \\
2 a^{2} s_{1}+6 a s_{2}+4 s_{3} & =q_{2,2}(a)=\frac{1}{6}\left(13 a^{2}+36 a+23\right) .
\end{aligned}
$$

Since $s_{0}=L+1$, this system permits us to compute $s_{1}, s_{2}, s_{3}$ as functions of $a=a_{L}$. For $L \geq 4$ we can arbitrarily fix $a$, and then we can obtain $a_{1}, \ldots, a_{L-1}$ from the values for $s_{1}, s_{2}, s_{3}$. Here, we are interested in the case $L=3$. In this situation, we obtain

$$
\begin{aligned}
-a_{1}-a_{2}+1 & =s_{1}=\frac{1}{12}\left(6 a^{2}-30 a+13\right) \\
a_{1}^{2}+a_{2}^{2}+1 & =s_{2}=\frac{1}{6}\left(-4 a^{3}+15 a^{2}+6\right) \\
-a_{1}^{3}-a_{2}^{3}+1 & =s_{3}=\frac{1}{24}\left(18 a^{4}-60 a^{3}+23\right) .
\end{aligned}
$$

With the aim of obtaining a polynomial equation for $a=a_{3}$, we compute

$$
\begin{aligned}
s_{1}^{2} & =a_{1}^{2}+a_{2}^{2}+1+2 a_{1} a_{2}-2 a_{1}-2 a_{2}=2 a_{1} a_{2}+s_{2}+2\left(s_{1}-1\right) \\
s_{1} s_{2} & =\ldots=s_{3}+\left(a_{1} a_{2}+1\right)\left(s_{1}-1\right)+s_{2}-1 .
\end{aligned}
$$

Elimination of the term $a_{1} a_{2}$ gives the relation

$$
s_{1}^{3}+2 s_{3}-3 s_{1} s_{2}-3 s_{1}^{2}+6 s_{1}+3 s_{2}-6=0
$$




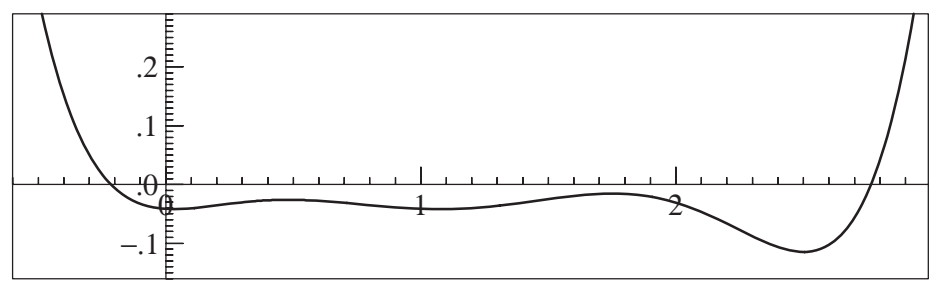

FIG. 2. The graph of the polynomial $\chi(a)$ of (4.3).

and leads to the polynomial equation for $a=a_{3}$

$$
\chi(a)=216 a^{6}-1512 a^{5}+3780 a^{4}-4032 a^{3}+1638 a^{2}-90 a-143=0 .
$$

A plot of this polynomial function is given in Fig. 2. There are two real zeros. One is close to -0.2144 and the other is (up to 20 digits)

$$
a_{3}=2.76487231590828234366 \text {. }
$$

This zero is in agreement with the numerical computations of Section 4.1.

\subsection{Order 4 is not possible}

Let us try to construct a differentiation matrix of the form (2.6), which has order $P=4$. By choosing $L \geq 4$ we can introduce more parameters. In addition to (4.1), there are three more order conditions for $P=4$. The critical one is for $(j, k)=(1,3)$ and it is given by

$$
\begin{array}{r}
2 A^{2} s_{1}+2 A\left(3 s_{2}-4 h s_{3}\right)+\left(4 s_{3}-10 h s_{4}+6 h^{2} s_{5}\right)=q_{1,3}^{h}(A) \\
=\frac{1}{6}\left(13 A^{2}+36 A+25-60 h-42 h A+35 h^{2}\right),
\end{array}
$$

where $A=a_{L}\left(1+a_{L} h\right)$. The left-hand side is the same as that for the last equation of (4.1). Therefore, we can have a differentiation matrix of order $P=4$ only if

$$
0=q_{1,3}^{h}(A)-q_{2,2}^{h}(A)=\frac{1}{6}\left(2+8 h A-2 h^{2}\right)
$$

(see Remark 3.4 of Section 3.3). This relation cannot be satisfied with $a_{L}=a_{L}(h)$ uniformly bounded for $h \rightarrow 0$.

\section{An alternative approach for order 4}

Up to an error of size $\mathcal{O}\left(h^{6}\right)$, we have

$$
h^{5} u^{(5)}(0) \approx \frac{1}{240}(-u(-3 h)+4 u(-2 h)-5 u(-h)+5 u(h)-4 u(2 h)+u(3 h))
$$


We keep the grid (2.1) unchanged, but consider differentiation matrices as in Section 2.2, where

$$
\mathcal{D}_{m, k}=\left\{\begin{aligned}
-\gamma /(240 h) & \text { if } k=m-3 \\
1 /(12 h)+4 \gamma /(240 h) & \text { if } k=m-2 \\
-8 /(12 h)-5 \gamma /(240 h) & \text { if } k=m-1 \\
0 & \text { if } k=m \\
8 /(12 h)+5 \gamma /(240 h) & \text { if } k=m+1 \\
-1 /(12 h)-4 \gamma /(240 h) & \text { if } k=m+2 \\
\gamma /(240 h) & \text { if } k=m+3
\end{aligned}\right.
$$

for indices $m, k$ satisfying $M \leq m \leq N-M$ or $M \leq k \leq N-M$ and for a given parameter $\gamma$. The bandwidth is increased by one, so that $R=3$. The underlying differentiation rule is of order 4 for all values of $\gamma$. For $\gamma=0$, we obtain the matrix of Section 4.1, and for $\gamma=4+\mathcal{O}\left(h^{2}\right)$ the coefficients of (5.1) represent a derivative approximation of order 6.

\subsection{Order conditions}

The matrix of the linear system (3.1) representing the order conditions remains the same, only the inhomogeneity vector $\mathbf{b}_{L, M}$ has to be adapted. Since the bandwidth of the matrix is increased, we work with $M=3$. With $s_{k}$ given by (3.6) and the notation $A=a_{L}\left(1+a_{L} h\right)$ in place, the condition (3.7) for order $P=4$ becomes

$$
\begin{aligned}
2 A\left(s_{0}-\right. & \left.2 h s_{1}\right)+\left(2 s_{1}-6 h s_{2}+4 h^{2} s_{3}\right)=q_{1,1}^{h}(A) \\
= & \frac{1}{6}\left(6 A^{2}+30 A+37-180 h-74 h A+215 h^{2}\right)+\frac{\gamma}{30} h^{2}, \\
A^{2} s_{0}+ & 2 A\left(2 s_{1}-3 h s_{2}\right)+\left(3 s_{2}-8 h s_{3}+5 h^{2} s_{4}\right)=q_{1,2}^{h}(A) \\
= & \frac{1}{6}\left(15 A^{2}+74 A+90-432 h-180 h A+510 h^{2}\right)+\frac{\gamma}{60} h, \\
2 A^{2} s_{1}+ & 2 A\left(3 s_{2}-4 h s_{3}\right)+\left(4 s_{3}-10 h s_{4}+6 h^{2} s_{5}\right)=q_{2,2}^{h}(A) \\
= & \frac{1}{6}\left(37 A^{2}+180 A+215-1020 h-434 h A+1189 h^{2}\right) \\
& +\frac{\gamma}{30}\left(1+3 h A-3 h^{2}\right), \\
2 A^{2} s_{1}+ & 2 A\left(3 s_{2}-4 h s_{3}\right)+\left(4 s_{3}-10 h s_{4}+6 h^{2} s_{5}\right)=q_{1,3}^{h}(A) \\
= & \frac{1}{6}\left(37 A^{2}+180 A+217-1020 h-426 h A+1187 h^{2}\right) \\
& -\frac{\gamma}{60}\left(3+14 h A-8 h^{2}\right), \\
3 A^{2} s_{2}+ & 2 A\left(4 s_{3}-5 h s_{4}\right)+\left(5 s_{4}-12 h s_{5}+7 h^{2} s_{6}\right)=q_{2,3}^{h}(A) \\
= & \left(15 A^{2}+72 A+85-396 h-170 h A+455 h^{2}\right)-\frac{\gamma}{60}(A+2 h), \\
4 A^{2} s_{3}+ & 2 A\left(5 s_{4}-6 h s_{5}\right)+\left(6 s_{5}-14 h s_{6}+8 h^{2} s_{7}\right)=q_{3,3}^{h}(A) \\
= & \frac{1}{6}\left(215 A^{2}+1020 A+1189-5460 h-2374 h A+6191 h^{2}\right) \\
& -\frac{\gamma}{30}\left(3-A^{2}+8 h A-7 h^{2}\right) .
\end{aligned}
$$




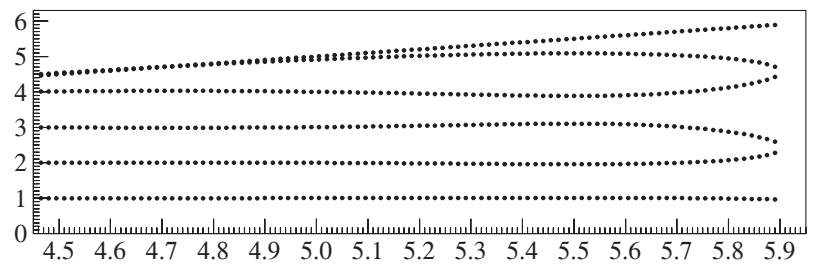

FIG. 3. The grid points $a_{1}, \ldots, a_{6}$ (top to bottom) as a function of $a_{6}$.

Since the left-hand expression is the same for the third and fourth equations, a necessary condition for order $P=4$ is that $q_{1,3}^{h}(A)=q_{2,2}^{h}(A)$. This condition yields a linear equation for $\gamma$, which gives

$$
\gamma=4 \cdot \frac{1+4 h A-h^{2}}{1+4 h A-\frac{14}{5} h^{2}}=4+\frac{36}{5} h^{2}-\frac{144 A}{5} h^{3}+\mathcal{O}\left(h^{4}\right) .
$$

It is interesting to note that with this choice of $\gamma$ we have, away from the end points, an order 6 approximation of the derivative.

Before passing to concrete computations, let us consider the limit case $h \rightarrow 0$.

\subsection{The limit case: $h \rightarrow 0$}

In the limit $h \rightarrow 0$ we see from (5.3) that $\gamma=4$. With this value of $\gamma$ the system (5.2) becomes (again we write $a$ for $a_{L}$ )

$$
\begin{aligned}
2 a s_{0}+2 s_{1} & =q_{1,1}(a)=\frac{1}{6}\left(6 a^{2}+30 a+37\right) \\
a^{2} s_{0}+4 a s_{1}+3 s_{2} & =q_{1,2}(a)=\frac{1}{6}\left(15 a^{2}+74 a+90\right) \\
2 a^{2} s_{1}+6 a s_{2}+4 s_{3} & =q_{2,2}(a)=\frac{1}{30}\left(185 a^{2}+900 a+1079\right) \\
2 a^{2} s_{1}+6 a s_{2}+4 s_{3} & =q_{1,3}(a)=\frac{1}{30}\left(185 a^{2}+900 a+1079\right) \\
3 a^{2} s_{2}+8 a s_{3}+5 s_{4} & =q_{2,3}(a)=\frac{1}{15}\left(225 a^{2}+1079 a+1275\right) \\
4 a^{2} s_{3}+10 a s_{4}+6 s_{5} & =q_{3,3}(a)=\frac{1}{30}\left(1079 a^{2}+5100 a+5933\right) .
\end{aligned}
$$

This system permits us to compute explicitly the expressions $s_{1}, \ldots, s_{5}$. We fix $L=6, M=3$ (so that $s_{0}=L+M-1=8$ ), choose arbitrarily $a_{L}=a_{6}$ and compute $a_{1}, \ldots, a_{5}$ from the nonlinear system given by $s_{1}, \ldots, s_{5}$. Figure 3 shows the numerically computed values $a_{1}, \ldots, a_{6}$ as function of $a_{6}$. For the parameter $a_{6}$, values between 4 and 6 are considered. If $a_{6}$ is too close to 6 , we have bad convergence of the Newton iterations. For $a_{6} \leq 5$, the grid points $a_{5}$ and $a_{6}$ are very close. The best choice of $a_{6}$ seems to be in the range between 5.7 and 5.8 .

\subsection{Numerical computations for the general case}

Encouraged by the successful computations for the limit case $h \rightarrow 0$ (or $N \rightarrow \infty$ ), we attack the general case with $h>0$. We fix $a_{L}=a_{6}=5.75$ and consider many different values of $N$ (recall that $h=1 / N$ ). The solution can be found numerically by Newton's method. Figure 4 shows the solution $a_{1}, \ldots, a_{6}$ as a 


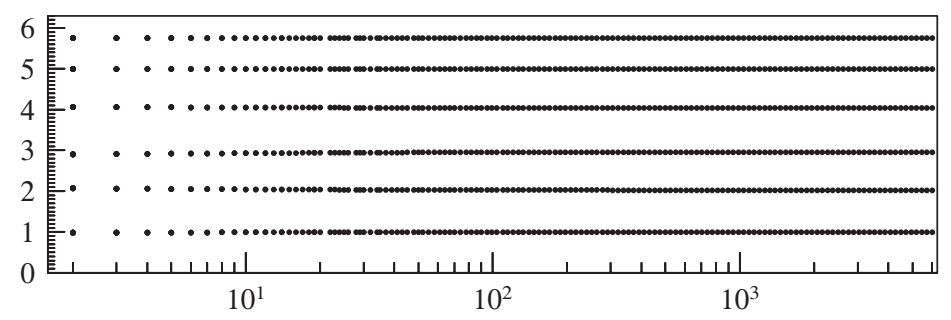

FIG. 4. The grid points $a_{1}, \ldots, a_{6}$ as a function of the parameter $N$ for the choice $a_{6}=5.75$.

function of $N$. It comes as a welcome surprise that the grid remains nearly unchanged for all values of $N$. For $N=1000$, we obtain

\begin{tabular}{c|ccc}
\hline$N$ & $a_{1} / a_{4}$ & $a_{2} / a_{5}$ & $a_{3} / a_{6}$ \\
\hline \multirow{2}{*}{1000} & 0.99533846829173 & 2.02375529528507 & 2.95622105838923 \\
& 4.03687073420997 & 4.99823111049117 & 5.75000000000000 \\
\hline
\end{tabular}

We see that the values $a_{1}, \ldots, a_{5}$ are very close to the continuation of the grid with constant step size. Similarly to the situation in Section 4.1, only the final subinterval is smaller.

The corresponding entries $\mathcal{D}_{j, k}$ of the differentiation matrix are obtained from the solution of a linear system (see Section 3.1). We have $(P-1) K=24$ equations for $K(K-1) / 2=28$ unknowns. This gives the freedom to fix some of the entries. To get a small bandwidth also in the left upper part (and the right lower part), we arbitrarily require

$$
\mathcal{D}_{j, k}=0 \quad \text { for } \quad|k-j| \geq 6 .
$$

There remain 25 unknowns for the 24 equations. We compute a least-squares solution from the QR decomposition of the relevant matrix. For $N=1000$, the leading digits of the coefficients $\mathcal{D}_{j, k}$, multiplied by $h$, are displayed in the following table:

\begin{tabular}{c|rrrrrrr}
\hline$h \mathcal{D}_{j, k}$ & $k=-5$ & $k=-4$ & $k=-3$ & $k=-2$ & $k=-1$ & $k=0$ & $k=1$ \\
\hline$j=-4$ & -0.385 & & & & & & \\
$j=-3$ & -0.323 & -0.255 & & & & & \\
$j=-2$ & 0.013 & -0.201 & -0.202 & & & & \\
$j=-1$ & 0.210 & -0.058 & -0.281 & -0.174 & & & \\
$j=0$ & -0.084 & 0.099 & -0.094 & -0.180 & -0.167 & & \\
$j=1$ & 0 & -0.019 & 0.238 & -0.082 & -0.343 & -0.324 & \\
$j=2$ & 0 & 0 & -0.085 & 0.067 & 0.116 & -0.037 & -0.686 \\
\hline
\end{tabular}




\section{Higher order differentiation matrices}

There does not seem to be serious difficulty in constructing skew-symmetric differentiation matrices of order higher than four. The construction of a $P$ th order skew-symmetric differentiation matrix follows the following steps:

Step 1 (Only for $P \geq 4$ ) Solve the $\left(\begin{array}{c}P-2 \\ 2\end{array}\right)$ compatibility conditions $Q_{j+1, k}^{h}(A)=Q_{j, k+1}^{h}(A), \quad 1 \leq j \leq$ $P-3, j+1 \leq k \leq P-2$ for $A=a_{L}\left(1+h a_{L}\right)$ and the basic $P$ th order differentiation rule; this step fixes $R$.

Step 2 If the compatibility conditions (step 1) are satisfied, the conditions (3.7) consist of $2 P-3$ nonlinear equations for $a_{1}, \ldots, a_{L}$, which can be solved by Newton's method; one typically keeps $a_{L}$ as a free parameter and one chooses $L=2 P-2$.

Step 3 Once the grid is fixed, the coefficients $\mathcal{D}_{j, k}$ of the differentiation matrix are obtained as a least square solution of the linear system $\mathcal{A}_{L, R} \mathcal{D}_{L, R}=\mathbf{b}_{L, R}$.

Let us shortly comment on these steps. For order $P=5$, the three compatibility conditions (step 1) are not fulfilled with the sixth-order basic differentiation formula (2.4). It turns out that a basic differentiation formula, satisfying the compatibility conditions, requires $R \geq 5$. One can take the scheme (2.4) of order 10. Although the number of compatibility conditions increases rapidly for $P>5$, they can all be satisfied (independent of the value of $A$ ) by the basic scheme (2.4) with $R=P$. This can be proved rigorously with the ideas of the proof of Lemma 2.2 in Kreiss \& Scherer (1974).

Step 2 is the critical one, because it is concerned with the solution of a nonlinear system of equations. We consider $a_{L}$ as a fixed parameter and choose $L=2 P-2$, so that the number of equations equals the number of unknowns $a_{1}, \ldots, a_{L-1}$. The leading digits of the numerically obtained coefficients are given (for $N=1000)$ in the following table for $P=5\left(a_{L}=7.65\right)$ and $P=6\left(a_{L}=9.70\right)$ :

\begin{tabular}{c|cccccccccc}
\hline & $a_{1}$ & $a_{2}$ & $a_{3}$ & $a_{4}$ & $a_{5}$ & $a_{6}$ & $a_{7}$ & $a_{8}$ & $a_{9}$ & $a_{10}$ \\
\hline$P=5$ & 1.00 & 1.98 & 3.07 & 3.88 & 5.14 & 5.89 & 7.07 & 7.65 & & \\
$P=6$ & 1.00 & 2.01 & 2.97 & 4.08 & 4.90 & 6.08 & 6.97 & 7.99 & 9.03 & 9.70 \\
\hline
\end{tabular}

Similarly, as for the case of orders $P=3$ and $P=4$, the grids are close to equidistant with the exception of the outermost interval $\left[-a_{L} h,-a_{L-1} h\right]$. Figure 5 shows the points $-a_{k} h$ for $k=1, \ldots, L$ (circles that are white inside) for the orders $P=3, P=4, P=5$ and $P=6$. Part of the equidistant grid $0, h, 2 h, 3 h, \ldots$ is shown by circles that are black inside. The vertical dotted lines indicate places for a grid that is equidistant everywhere.

Step 3 is straightforward as the solution of a linear system. There are fewer equations than unknowns. Similar as for $P=4$, one can use this freedom to make zero some elements that are far away from the diagonal and to minimize the modulus of the elements $\mathcal{D}_{j, k}$.

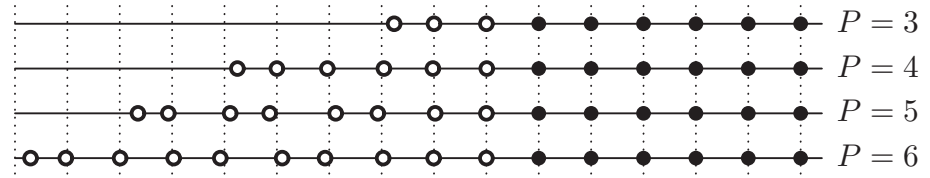

FIG. 5. Distribution of the grid points at the left end of the interval. 


\section{Funding}

This work was partially supported by the Fonds National Suisse, Project No. 200020_159856.

\section{REFERENCES}

Hairer, E. \& Iserles, A. (2016) Numerical stability in the presence of variable coefficients. Found. Comput. Math., 16, 751-777.

IsERLES, A. (2014) On skew-symmetric differentiation matrices. IMA J. Numer. Anal., 34, 435-451.

Kitson, A., MCLACHLAN, R. I. \& RoBiDOuX, N. (2003) Skew-adjoint finite difference methods on nonuniform grids. New Zealand J. Math., 32, 139-159.

KREISS, H.-O. \& SCHERER, G. (1974) Finite element and finite difference methods for hyperbolic partial differential equations. Proceedings of a Symposium conducted by the Mathematics Research Center (C. de Boor ed.), University of Wisconsin, Madison, WI, April 1-3, 1974. London: Academic Press, pp. 195-212.

MCLACHLAN, R. I. (2003) Spatial discretization of partial differential equations with integrals. IMA J. Numer. Anal., 23, 645-664.

Olsson, P. (1995) Summation by parts, projections, and stability. I. Math. Comp., 64, 1035-1065, S23-S26.

STRAND, B. (1994) Summation by parts for finite difference approximations for $d / d x$. J. Comput. Phys., 110, 47-67. 